\title{
Structural and spectral properties of 4-phenoxyphthalonitrile dye sensitizer for solar cell applications
}

\author{
P M ANBARASAN*，K VASUDEVAN，P SENTHIL KUMAR，A PRAKASAM，M GEETHA \\ and K LALITHAMBIGAI \\ Department of Physics, Periyar University, Salem 636 011, India
}

MS received 22 December 2010; revised 22 February 2011

\begin{abstract}
The geometries, electronic structures, polarizabilities and hyperpolarizabilities of organic dye sensitizer 4-phenoxyphthalonitrile was studied based on ab initio HF and density functional theory (DFT) using the hybrid functional B3LYP. Ultraviolet-visible (UV-Vis) spectrum was investigated by time dependent DFT (TDDFT). Features of the electronic absorption spectrum in the visible and near-UV regions were assigned based on TD-DFT calculations. The absorption bands were assigned to $\pi \rightarrow \pi^{*}$ transitions. Calculated results suggest that the three excited states with the lowest excited energies in 4-phenoxyphthalonitrile was due to photo-induced electron transfer processes. The interfacial electron transfer between semiconductor $\mathrm{TiO}_{2}$ electrode and dye sensitizer 4-phenoxyphthalonitrile was due to an electron injection process from excited dye to the semiconductor's conduction band. The role of phenoxy group in 4-phenoxyphthalonitrile in geometries, electronic structures and spectral properties were analysed.
\end{abstract}

Keywords. Electronic structure; absorption spectrum; dye sensitizer; density functional theory; vibrational spectra.

\section{Introduction}

The new technologies for direct solar energy conversion have gained more attention in the last few years. In particular, Dye sensitized solar cells (DSSCs) are promising in terms of efficiency and low cost (Regan and Gratzel 1991; Gratzel 2001; Park and Kim 2008). The foremost feature of DSSC is that it consists of a wide bandgap nanocrystalline film grafted with a quasi-monolayer of dye molecules and submerged in a redox electrolyte. This elegant architecture can synchronously address two critical issues of employing organic materials for the photovoltaic applications: (i) efficient charge generation from the Frenkel excitons; (ii) longlived electron-hole separation up to the millisecond time domain. The latter attribute can often confer an almost quantitative charge collection for several micrometer-thick active layers, even if the electron mobilities in nanostructured semiconducting films are significantly lower than those in the bulk crystalline materials. Benefitted from systematic device engineering and continuous material innovation, a state of the art DSSC with a ruthenium sensitizer has achieved a validated efficiency of $11.1 \%$ (Chiba et al 2006) measured under the air mass 1.5 global (AM1.5G) conditions. In view of the limited ruthenium resource and the heavy-metal toxicity, metal-free organic dyes have received surging research interest in recent years (Hara et al 2003a, b; Campbell et al 2004; Horiuchi et al 2004; Kitamura et al 2004; Thomas et al 2005; Hagberg et

\footnotetext{
*Author for correspondence (anbarasanpm@gmail.com)
}

al 2006; Li et al 2006; Kim et al 2006; Koumura et al 2006; Edvinsson et al 2007; Wang et al 2007, 2008; Shi et al 2008; Zhou et al 2008; Lin et al 2009; Xu et al 2009; Zhang et al 2009a, b, c. Because of their high molar absorption coefficient, relatively simple synthesis procedure, various structures and lower cost in contrast to a ruthenium dye and the flexibility in molecular tailoring of an organic sensitizer provides a large area to explore (Liang et al 2007; Zhang et al 2007; Xu et al 2008). Meanwhile, recently a rapid progress of organic dyes has been witnessed reaching close to $10.0 \%$ efficiencies in combination with a volatile acetonitrile-based electrolyte (Ito et al 2008). Phthalonitrile is an important class of high-performance dyes, which are easily processable, and display good mechanical properties, outstanding thermal and thermal-oxidative stability. Phthalonitrile dyes were used for aerospace, marine and electronic packaging applications. Thermal treatment of phthalonitrile derivatives was carried out at elevated temperatures (generally high up to $350^{\circ} \mathrm{C}$ ) for an extended period of time. In this paper, the performance of 4-phenoxyphthalonitrile metal free dye that was used in DSSC is analysed.

\section{Experimental}

The compound, 4-phenoxyphthalonitrile, was obtained from Sigma-Aldrich Chemical Company, USA, with a purity of greater than $99 \%$ and it was used as such without further purification. The FT-Raman spectrum of 4phenoxyphthalonitrile was recorded using $1064 \mathrm{~nm}$ line of 
Nd:YAG laser as excitation wavelength in the region 50 $3500 \mathrm{~cm}^{-1}$ on a Brucker model IFS $66 \mathrm{~V}$ spectrophotometer. The FT-IR spectrum of this compound was recorded in the region $400-4000 \mathrm{~cm}^{-1}$ on IFS $66 \mathrm{~V}$ spectrophotometer using $\mathrm{KBr}$ pellet technique. The spectrum was recorded at room temperature, with a scanning speed of $30 \mathrm{~cm}^{-1} \mathrm{~min}^{-1}$ and a spectral resolution of $2 \mathrm{~cm}^{-1}$.

\section{Computational methods}

The computations of the geometries, electronic structures, polarizabilities and hyperpolarizabilities, as well as electronic absorption spectrum for dye sensitizer 4phenoxyphthalonitrile was done using ab initio $\mathrm{HF}$ and DFT with Gaussian03 package (Frisch et al 2003). The DFT was treated according to Becke's three parameter gradient-corrected exchange potential and the Lee-YangParr gradient-corrected correlation potential (B3LYP) (Lee et al 1988; Miehlich et al 1989; Becke 1993), and all calculations were performed without any symmetry constraints by using polarized split-valence $6-311 \mathrm{G}(\mathrm{d}, \mathrm{p})$ basis sets. The electronic absorption spectrum requires calculation of the allowed excitations and oscillator strengths. These calculations were done using TD-DFT with the same basis sets and exchange-correlation functional in vacuum and solution, and the non-equilibrium version of the polarizable continuum model (PCM) (Barone and Cossi 1998; Cossi et al 2003) was adopted for calculating the solvent effects.

\section{Results and discussion}

\subsection{Geometric structure}

The optimized geometry of the 4-phenoxyphthalonitrile is shown in figure 1 , and the bond lengths, bond angles and dihedral angles are listed in table 1 . Since the crystal structure of the exact title compound is not available until now, the

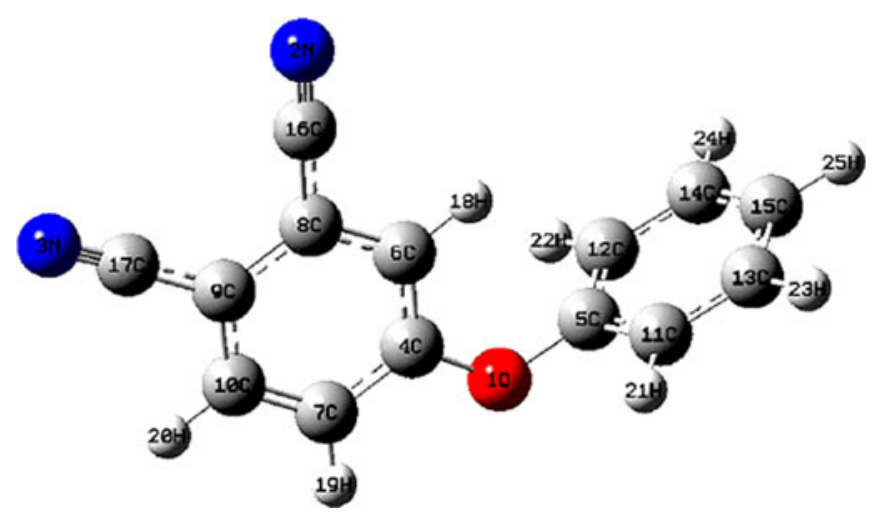

Figure 1. Optimized geometrical structure of dye 4-phenoxyphthalonitrile.
Table 1. Bond lengths (in $\AA$ ), bond angles (in degree) and dihedral angles (in degree) of the dye 4-phenoxyphthalonitrile.

\begin{tabular}{lll}
\hline Parameters & HF/6-311G (d,p) $\quad$ B3LYP/6-311G (d,p) \\
\hline
\end{tabular}

Bond length $(\AA)$

O1-C4

$1 \cdot 3447$

$1 \cdot 3615$

$\mathrm{O} 1-\mathrm{C} 5$

1.377

$1 \cdot 3975$

N2-C16

$1 \cdot 1293$

$1 \cdot 1545$

N3-C17

1.1299

$1 \cdot 1551$

1.3847

1.3946

C4-C6

1.3906

1.4003

C5-C11

1.3805

1.3902

C5-C12

C6-C8

C6-H18

$1 \cdot 3805$

1.3902

1.3987

$1 \cdot 3878$

1.0807

C7-C10

1.3742

1.3826

$1.0733 \quad 1.0825$

$1.3906 \quad 1.4104$

$\begin{array}{ll}1.4432 & 1.4307\end{array}$

C8-C9

$\mathrm{C} 8-\mathrm{C} 16$

1.3916

1.4031

1.4391

1.4270

C9-C17

C10-H20

C11-C13

1.0736

1.0825

1.3934

C11-H21

1.3849

1.0833

1.3934

1.3849

1.0833

C12-H22

C13-C15

C13-H23

1.0746

1.3938

1.0837

1.0749

1.385

1.3938

C14-H24

C15-H25

1.0749

1.0837

1.0835

Bond angle $\left(^{\circ}\right)$

C4-O1-C5

1.0747

120.0163

121.2095

123.9884

O1-C4-C6

124.1427

$115 \cdot 8781$

$120 \cdot 1335$

120.0822

119.1878

119.2758

119. 1795

O1-C5-C11

119.2714

121.5417

121.394

119.699

C4-C6-C8

119.4028

$121 \cdot 1244$

120.8084

119.4728

119.4926

$120 \cdot 1108 \quad 120 \cdot 0935$

118.7329

118.6707

$\begin{array}{ll}121.1564 & 121.2358\end{array}$

$\begin{array}{ll}120 \cdot 9184 & 120 \cdot 4914\end{array}$

$118.0395 \quad 118.5478$

121.0421

$120 \cdot 9609$

118.792

121.797

118.6867

121.5693

119.744

119.4109

$120 \cdot 8959$

C7-C10-C9

$120 \cdot 6938$

$120 \cdot 0719$

$120 \cdot 0314$

119.0323

C9-C10-H20

119.2748

$119 \cdot 0767$

optimized structure can only be compared with other similar systems for which the crystal structures were solved. From the theoretical values we can find that most of the optimized 
Table 1. (contd.)

\begin{tabular}{|c|c|c|}
\hline Parameters & $\mathrm{HF} / 6-311 \mathrm{G}(\mathrm{d}, \mathrm{p})$ & B3LYP/6-311G (d,p) \\
\hline \multicolumn{3}{|l|}{ Bond angle $\left({ }^{\circ}\right)$} \\
\hline $\mathrm{C} 5-\mathrm{C} 11-\mathrm{H} 21$ & $119 \cdot 6654$ & $119 \cdot 6143$ \\
\hline C13-C11-H21 & $121 \cdot 256$ & $121 \cdot 412$ \\
\hline C5-C12-C14 & $119 \cdot 0774$ & $118 \cdot 9721$ \\
\hline C5-C12-H22 & $119 \cdot 663$ & $119 \cdot 6124$ \\
\hline C14-C12-H22 & $121 \cdot 2577$ & 121.4126 \\
\hline C11-C13-C15 & $120 \cdot 3044$ & $120 \cdot 2689$ \\
\hline C11-C13-H23 & $119 \cdot 5794$ & $119 \cdot 5803$ \\
\hline C15-C13-H23 & $120 \cdot 1159$ & $120 \cdot 1506$ \\
\hline C12-C14-C15 & $120 \cdot 3038$ & $120 \cdot 2674$ \\
\hline C12-C14-H24 & $119 \cdot 5799$ & $119 \cdot 5797$ \\
\hline C15-C14-H24 & $120 \cdot 1161$ & $120 \cdot 1527$ \\
\hline C13-C15-C14 & $119 \cdot 8417$ & $119 \cdot 978$ \\
\hline C13-C15-H25 & $120 \cdot 0784$ & $120 \cdot 0089$ \\
\hline $\mathrm{C} 14-\mathrm{C} 15-\mathrm{H} 25$ & $120 \cdot 0791$ & $120 \cdot 0121$ \\
\hline \multicolumn{3}{|l|}{ Dihedral angle $\left(^{\circ}\right)$} \\
\hline C5-O1-C4-C6 & -0.0362 & $0 \cdot 0133$ \\
\hline $\mathrm{C} 5-\mathrm{O} 1-\mathrm{C} 4-\mathrm{C} 7$ & $179 \cdot 9662$ & -179.9876 \\
\hline C4-O1-C5-C11 & $91 \cdot 3854$ & 91.6632 \\
\hline $\mathrm{C} 4-\mathrm{O} 1-\mathrm{C} 5-\mathrm{C} 12$ & $-91 \cdot 3633$ & -91.7513 \\
\hline $\mathrm{O} 1-\mathrm{C} 4-\mathrm{C} 6-\mathrm{C} 8$ & $-179 \cdot 9947$ & $-180 \cdot 0011$ \\
\hline O1-C4-C6-H18 & 0.0047 & -0.0038 \\
\hline C7-C4-C6-C8 & $0 \cdot 0028$ & $-0 \cdot 0002$ \\
\hline C7-C4-C6-H18 & -179.9978 & 179.9971 \\
\hline $\mathrm{O} 1-\mathrm{C} 4-\mathrm{C} 7-\mathrm{C} 10$ & $179 \cdot 9955$ & $180 \cdot 003$ \\
\hline O1-C4-C7-H19 & -0.0035 & 0.0037 \\
\hline C6-C4-C7-C10 & $-0 \cdot 0022$ & $0 \cdot 0022$ \\
\hline C6-C4-C7-H19 & $179 \cdot 9988$ & -179.9972 \\
\hline $\mathrm{O} 1-\mathrm{C} 5-\mathrm{C} 11-\mathrm{C} 13$ & $177 \cdot 6333$ & $176 \cdot 7791$ \\
\hline O1-C5-C11-H21 & $-1 \cdot 8763$ & $-2 \cdot 6059$ \\
\hline C12-C5-C11-C13 & 0.4423 & $0 \cdot 2773$ \\
\hline C12-C5-C11-H21 & $-179 \cdot 0674$ & $-179 \cdot 1078$ \\
\hline O1-C5-C12-C14 & $-177 \cdot 6334$ & $-176 \cdot 7794$ \\
\hline $\mathrm{O} 1-\mathrm{C} 5-\mathrm{C} 12-\mathrm{H} 22$ & 1.8769 & $2 \cdot 6065$ \\
\hline C11-C5-C12-C14 & -0.4423 & -0.2773 \\
\hline C11-C5-C12-H22 & $179 \cdot 0681$ & $179 \cdot 1087$ \\
\hline C4-C6-C8-C9 & -0.0016 & -0.0032 \\
\hline C4-C6-C8-C16 & 179.9958 & $179 \cdot 9935$ \\
\hline H18-C6-C8-C9 & 179.999 & $179 \cdot 9995$ \\
\hline H18-C6-C8-C16 & -0.0036 & -0.0037 \\
\hline C4-C7-C10-C9 & 0.0004 & -0.0008 \\
\hline C4-C7-C10-H20 & $180 \cdot 0001$ & 179.9993 \\
\hline H19-C7-C10-C9 & $-180 \cdot 0007$ & 179.9985 \\
\hline H19-C7-C10-H20 & -0.001 & -0.0014 \\
\hline C6-C8-C9-C10 & -0.0002 & 0.0045 \\
\hline C6-C8-C9-C17 & $179 \cdot 9976$ & $180 \cdot 002$ \\
\hline C16-C8-C9-C10 & -179.9975 & -179.9921 \\
\hline C16-C8-C9-C17 & 0.0003 & 0.0053 \\
\hline C8-C9-C10-C7 & 0.0009 & $-0 \cdot 0025$ \\
\hline C8-C9-C10-H20 & -179.9989 & $179 \cdot 9974$ \\
\hline C17-C9-C10-C7 & -179.997 & $-180 \cdot 0$ \\
\hline C17-C9-C10-H20 & 0.0033 & $-0 \cdot 0001$ \\
\hline
\end{tabular}

bond lengths, bond angles and dihedral angles. The optimized bond lengths of $\mathrm{C} 8-\mathrm{C} 16$ and $\mathrm{C} 9-\mathrm{C} 17$ is 1.4307 and $1.4270 \AA$, respectively at B3LYP/6-311G (d,p) and also well matched with HF/6-311G (d,p).
Table 1. (contd.)

\begin{tabular}{lcc}
\hline Parameters & HF/6-311G (d,p) & B3LYP/6-311G (d,p) \\
\hline Dihedral angle $\left.{ }^{\circ}\right)$ & & \\
C5-C11-C13-C15 & -0.0956 & -0.001 \\
C5-C11-C13-H23 & -179.9084 & -179.8365 \\
H21-C11-C13-C15 & 179.4059 & $179 \cdot 3726$ \\
H21-C11-C13-H23 & $-0 \cdot 4069$ & -0.463 \\
C5-C12-C14-C15 & 0.0956 & 0.0011 \\
C5-C12-C14-H24 & 179.9082 & $179 \cdot 8367$ \\
H22-C12-C14-C15 & -179.4067 & -179.3734 \\
H22-C12-C14-H24 & 0.406 & 0.4622 \\
C11-C13-C15-C14 & $-0 \cdot 2411$ & -0.2687 \\
C11-C13-C15-H25 & -179.9366 & -179.9195 \\
H23-C13-C15-C14 & 179.5707 & 179.5659 \\
H23-C13-C15-H25 & -0.1248 & -0.0849 \\
C12-C14-C15-C13 & $0 \cdot 2412$ & 0.2686 \\
C12-C14-C15-H25 & 179.9367 & 179.9194 \\
H24-C14-C15-C13 & -179.5705 & -179.566 \\
H24-C14-C15-H25 & $0 \cdot 125$ & 0.0848 \\
\hline
\end{tabular}

\subsection{Electronic structures and charges}

Natural bond orbital (NBO) analysis was performed in order to analyse the charge populations of the dye 4phenoxyphthalonitrile. Charge distributions in $\mathrm{C}, \mathrm{N}$ and $\mathrm{H}$ atoms were observed because of the different electronegativity, the electrons transferred from $\mathrm{C}$ atoms to $\mathrm{C}$, $\mathrm{N}$ atoms, $\mathrm{C}$ atoms to $\mathrm{H}$. The natural charges of different groups are the sum of every atomic natural charge in the group. These data indicate that the cyanine and amide groups are acceptors, while the acetic groups are donors, and the charges were transferred through chemical bonds. The frontier molecular orbitals (MO) energies and corresponding density of state of the dye 4-phenoxyphthalonitrile

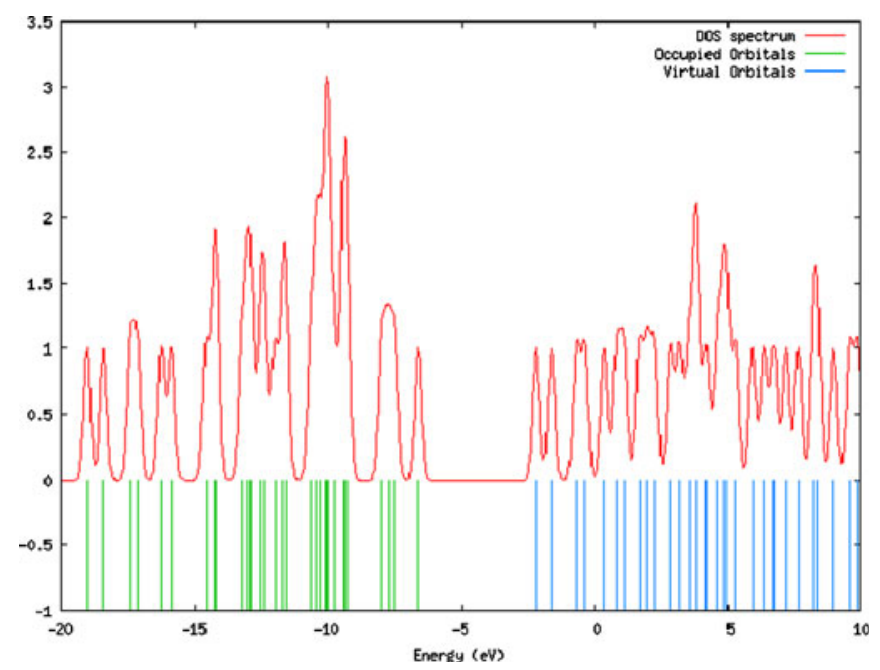

Figure 2. Frontier molecular orbital energies and corresponding density of state (DOS) spectrum of the dye 4-phenoxyphthalonitrile. 
is shown in figure 2. The HOMO-LUMO gap of the dye 4-phenoxyphthalonitrile in vacuum is $4.5952 \mathrm{eV}$.

While the calculated HOMO and LUMO energies of the bare $\mathrm{Ti}_{38} \mathrm{O}_{76}$ cluster as a model for nanocrystalline are -6.55 and $-2.77 \mathrm{eV}$, respectively resulting in a HOMO-LUMO gap of $3.78 \mathrm{eV}$, the lowest transition is reduced to $3.20 \mathrm{eV}$ according to TD-DFT, and this value is slightly smaller than typical bandgap of $\mathrm{TiO}_{2}$ nanoparticles with $\mathrm{nm}$ size (Nazeeruddin et al 2005). Furthermore, the HOMO, LUMO and HOMO-LUMO gap of $\left(\mathrm{TiO}_{2}\right)_{60}$ cluster is $-7.52,-2.97$ and $4.55 \mathrm{eV}$ (B3LYP/VDZ), respectively (Lundqvist et al 2006). Taking into account the cluster size effects and the calculated HOMO, LUMO, HOMO-LUMO gap of the dye 4-phenoxyphthalonitrile, $\mathrm{Ti}_{38} \mathrm{O}_{76}$ and $\left(\mathrm{TiO}_{2}\right)_{60}$ clusters, we can find that the HOMO energies of these dyes fall within the $\mathrm{TiO}_{2}$ gap.

The above data also reveal the interfacial electron transfer between semiconductor $\mathrm{TiO}_{2}$ electrode and the dye sensitizer. 4-Phenoxyphthalonitrile is the electron injection process from excited dye to the semiconductor conduction band. This is a kind of typical interfacial electron transfer reaction (Waston and Meyer 2005).

\subsection{Polarizability and hyperpolarizability}

Polarizabilities and hyperpolarizabilities characterize the response of a system in an applied electric field (Datta and Pati 2003, 2004, 2005a, b, 2006; Zhang et al 2004; Datta et al 2005, 2006; Datta 2009). They determine not only the strength of molecular interactions (long-range intermolecular induction, dispersion forces, etc) as well as the cross sections of different scattering and collision processes, but also the nonlinear optical properties (NLO) of the system (Christiansen et al 1999; Sun et al 2003). It was found that the dye sensitizer hemicyanine system with high NLO property, usually possesses high photoelectric conversion performance (Wang et al 2000). In order to investigate the relationships among photocurrent generation, molecular structures and NLO, the polarizabilities and hyperpolarizabilities of 4-phenoxyphthalonitrile was calculated.

The polarizabilities and hyperpolarizabilities were computed via finite field (FF) method, sum-over state (SOS) method based on TD-DFT and coupled-perturbed HF (CPHF) method. However, the use of FF, SOS and CPHF methods with large-sized basis sets for 4phenoxyphthalonitrile is too expensive. Here, the polarizability and the first hyperpolarizabilities are computed as a numerical derivative of the dipole moment using B3LYP/6-
31G (d,p). The definitions (Christiansen et al 1999; Sun et al 2003) for the isotropic polarizability is

$$
\alpha=\frac{1}{3}\left(\alpha_{X X}+\alpha_{Y Y}+\alpha_{Z Z}\right) .
$$

The polarizability anisotropy invariant is

$$
\Delta \alpha=\left[\frac{\left(\alpha_{X X}-\alpha_{Y Y}\right)^{2}+\left(\alpha_{Y Y}-\alpha_{Z Z}\right)^{2}+\left(\alpha_{Z Z}-\alpha_{X X}\right)^{2}}{2}\right]^{\frac{1}{2}},
$$

and the average hyperpolarizability is

$$
\beta_{0}=\frac{1}{5}\left(\beta_{i i Z}+\beta_{i Z i}+\beta_{Z i i}\right),
$$

where $\alpha_{X X}, \alpha_{Y Y}$ and $\alpha_{Z Z}$ are tensor components of polarizability; $\beta_{i i Z}, \beta_{i Z i}$ and $\beta_{Z i i}$ (i from $X$ to $Z$ ) are tensor components of hyperpolarizability.

Tables 2 and 3 list the values of the polarizabilities and hyperpolarizabilities of the dye 4-phenoxyphthalonitrile. In addition to the individual tensor components of the polarizabilities and the first hyperpolarizabilities, the isotropic polarizability, polarizability anisotropy invariant and hyperpolarizability are also calculated. The calculated isotropic polarizability of 4-phenoxyphthalonitrile is -53.6627 a.u. However, the calculated isotropic polarizability of JK16, JK17, dye 1, dye 2, D5, DST and DSS is 759.9, 1015.5, $694 \cdot 7,785 \cdot 7,510 \cdot 6,611.2$ and 802.9 a.u., respectively (Seidl et al 1996; Zhang et al 2009a, b, c). The above data indicate that the donor-conjugate $p$ bridge-acceptor (D-p-A) chainlike dyes have stronger response for external electric field. Whereas, for dye sensitizers D5, DST, DSS, JK16, JK17, dye 1 and dye 2, on the basis of the published photo-to-current conversion efficiencies, the similarity and the difference of geometries, and the calculated isotropic polarizabilities, it is found that longer the length of the conjugate bridge in similar dyes, the larger the polarizability of the dye molecule, and the lower the photo-to-current conversion efficiency. This may be due to the fact that the longer conjugate-p-bridge enlarged the delocalization of electrons, thus it enhanced the response of the external field, but the enlarged delocalization may be not favourable to generate charge separated state effectively. So it induces the lower photo-to-current conversion efficiency.

\subsection{IR and Raman frequencies}

Figures 3 and 4 show the observed and calculated IR and Raman spectra of 4-phenoxyphthalonitrile, respectively.

Table 2. Polarizability $(\alpha)$ of the dye 4-phenoxyphthalonitrile (in a.u.).

\begin{tabular}{lcccccr}
\hline$\alpha_{\mathrm{xx}}$ & $\alpha_{\mathrm{xy}}$ & $\alpha_{\mathrm{yy}}$ & $\alpha_{\mathrm{xz}}$ & $\alpha_{\mathrm{yz}}$ & $\alpha_{\mathrm{zz}}$ & $\alpha$ \\
\hline-117.2284 & 19.5787 & -104.4547 & 0.7541 & 0.0585 & -91.5667 & -53.6627 \\
\hline
\end{tabular}


Table 3. Hyperpolarizability ( $\beta$ ) of the dye 4-phenoxyphthalonitrile (in a.u.).

\begin{tabular}{lcccccccccc}
\hline$\beta_{\mathrm{xxx}}$ & $\beta_{\mathrm{xxy}}$ & $\beta_{\mathrm{xyy}}$ & $\beta_{\mathrm{yyy}}$ & $\beta_{\mathrm{xxz}}$ & $\beta_{\mathrm{xyz}}$ & $\beta_{\mathrm{yyz}}$ & $\beta_{\mathrm{xzz}}$ & $\beta_{\mathrm{yzz}}$ & $\beta_{\mathrm{zzz}}$ & $\beta_{\mathrm{ii}}$ \\
\hline 234.1205 & -17.4479 & 19.7551 & -68.7671 & 5.7017 & 0.3518 & 0.9628 & 23.1031 & 0.5898 & 2.1785 & 5.3058 \\
\hline
\end{tabular}

Comparison of the observed (FT-IR and FT-Raman) and calculated vibrational frequencies of 4-phenoxyphthalonitrile is shown in table 4. Comparison of the frequencies calculated by ab initio HF and B3LYP with experimental values reveals the overestimation of the calculated vibrational modes due to the neglect of anharmonicity in real system. Inclusion of electron correlation in density functional theory to a certain extent makes the frequency values smaller in comparison with experimental values. Anyway notwithstanding the level of calculations, it is customary to scale down the calculated harmonic frequencies in order to improve the agreement with the experiment. In our study we have followed two different scaling factors B3LYP/6-311G (d,p) and HF/6$311 \mathrm{G}(\mathrm{d}, \mathrm{p})$. The 4-phenoxyphthalonitrile molecule gives rise to thirty $\mathrm{C}-\mathrm{C}$ ring deformations at $15,33,92,143,251$, 303, 395, 404, 423, 431, 455, 497, 555, 559, 607, 629, 641, 649, 708, 714, 741, 746, 796, 844, 973, 981, 1019, 1094, 1107 and $1176 \mathrm{~cm}^{-1}$, two $\mathrm{C}-\mathrm{C}-\mathrm{C}$ inplane bending vibrations at 55 and $118 \mathrm{~cm}^{-1}$, eleven $\mathrm{C}-\mathrm{C}-\mathrm{N}$ inplane bending vibrations at 159, 242, 303, 455, 497, 559, 607, 649, 708,714 and $741 \mathrm{~cm}^{-1}$, twenty four $\mathrm{CH}$ wagging at 854 , 897, 930, 1042, 1094, 1107, 1176, 1182, 1187, 1219, 1225, 1268, 1302, 1322, 1344, 1441, 1485, 1518, 1524, 1595, 1627,1636 and $1644 \mathrm{~cm}^{-1}$, seven $\mathrm{C}=\mathrm{C}$ stretching vibrations at $741,973,1187,1219,1225,1268$ and $1302 \mathrm{~cm}^{-1}$, two $\mathrm{CH}$ out-of-plane bending vibrations at 796 and $844 \mathrm{~cm}^{-1}$, one $\mathrm{CO}$ stretching vibration at $850 \mathrm{~cm}^{-1}$, one $\mathrm{C}-\mathrm{H}$ torsion at $1006 \mathrm{~cm}^{-1}$, three $\mathrm{C}-\mathrm{C}-\mathrm{N}$ stretching vibrations at 1219 ,

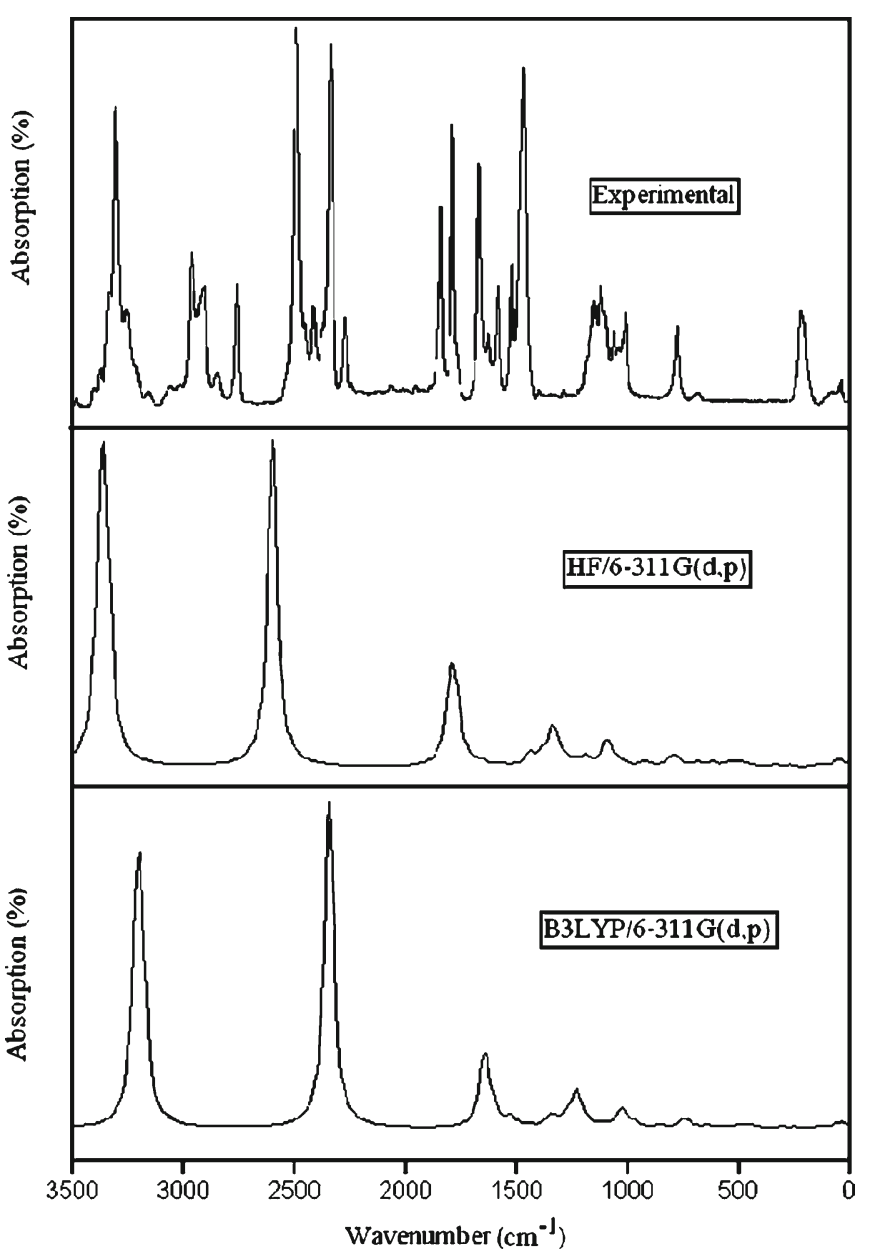

Figure 4. Observed and calculated FT-Raman spectra of 4-phenoxyphthalonitrile.
Figure 3. Observed and calculated FT-IR spectra of 4-phenoxyphthalonitrile. ryph ha lonitrile.

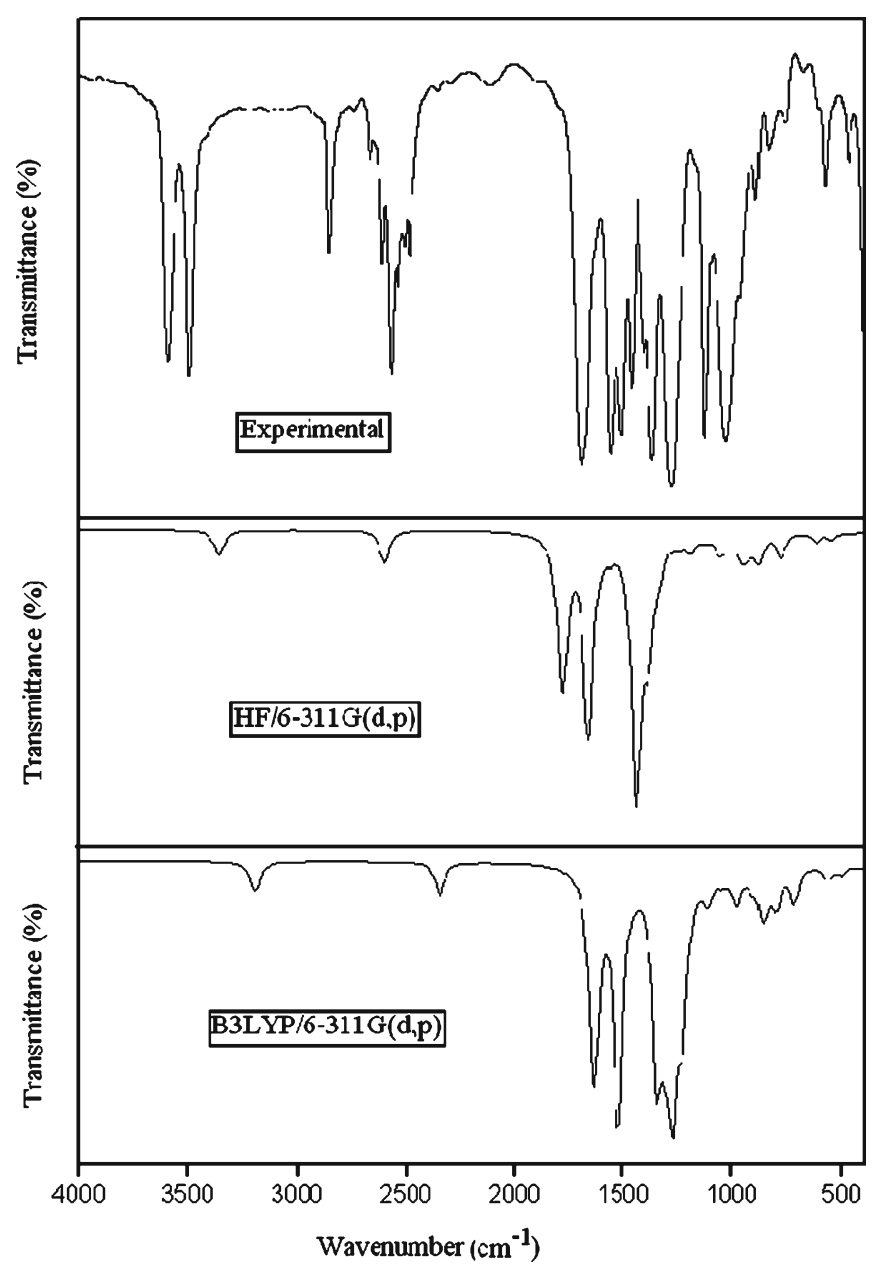




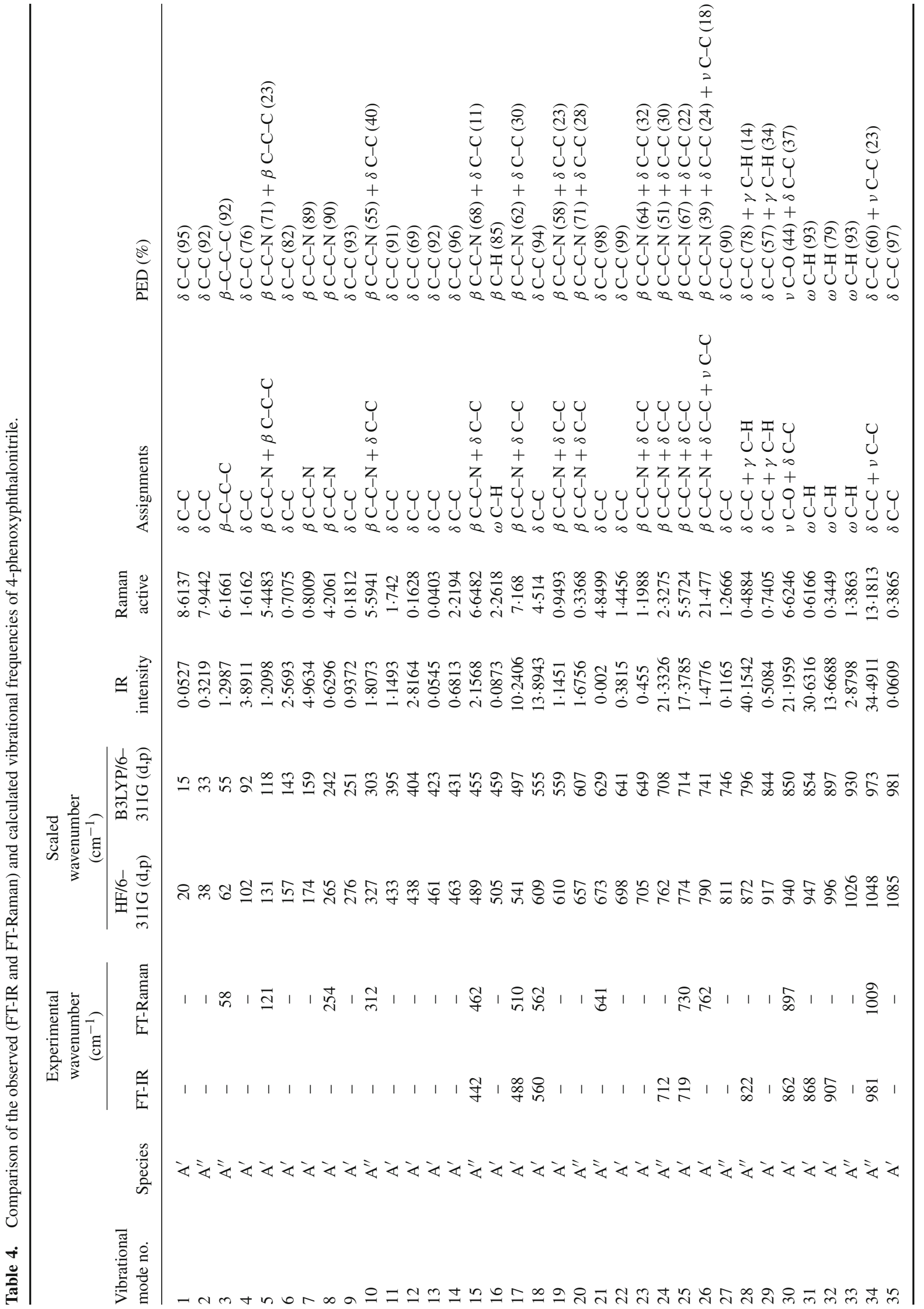




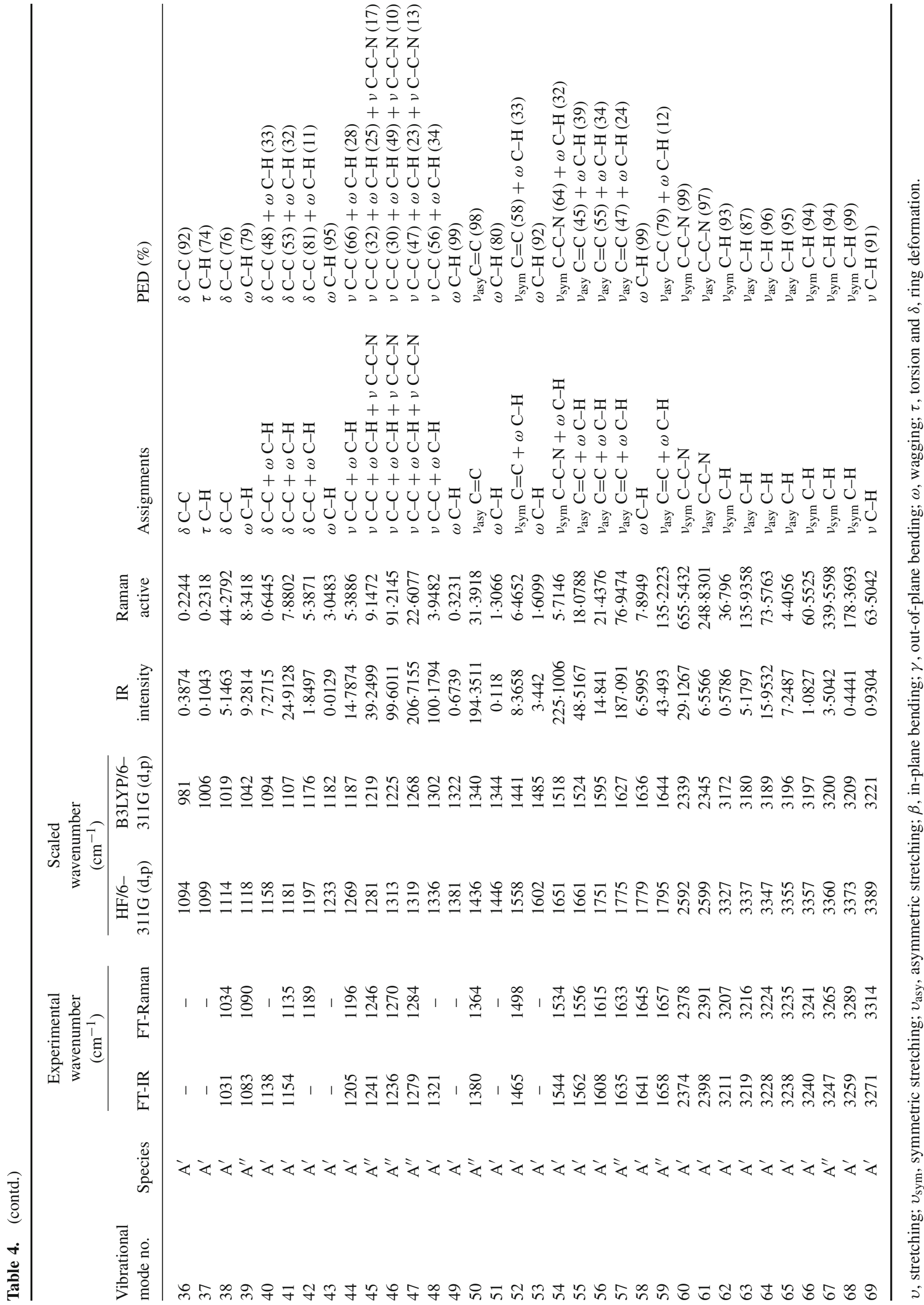


1225 and $1268 \mathrm{~cm}^{-1}$, five $\mathrm{C}=\mathrm{C}$ asymmetric stretching vibrations at $1340,1524,1595,1627$ and $1644 \mathrm{~cm}^{-1}$, one $\mathrm{C}=\mathrm{C}$ symmetric stretching vibration at $1441 \mathrm{~cm}^{-1}$, two $\mathrm{C}-\mathrm{C}-\mathrm{N}$ symmetric stretching vibrations at 1518 and $2339 \mathrm{~cm}^{-1}$, one $\mathrm{C}-\mathrm{C}-\mathrm{N}$ asymmetric stretching vibration at $2345 \mathrm{~cm}^{-1}$, four $\mathrm{C}-\mathrm{H}$ symmetric stretching vibrations at $3172,3196,3200$ and $3209 \mathrm{~cm}^{-1}$, three $\mathrm{C}-\mathrm{H}$ asymmetric stretching vibrations at 3180,3189 and $3196 \mathrm{~cm}^{-1}$ and one C-H stretching vibration at $3221 \mathrm{~cm}^{-1}$ were assigned to B3LYP/6-311G (d,p) scaling method. The next stronger IR absorption is attributed to vibrational mode 47 near about $1268 \mathrm{~cm}^{-1}$, corresponding to the stretching mode of $\mathrm{C}=\mathrm{C}$ bonds and stretching of $\mathrm{C}-\mathrm{C}-$ $\mathrm{N}$ bond. In the Raman spectrum, however, the strongest activity mode is the vibrational mode 60 near about $2339 \mathrm{~cm}^{-1}$, which is corresponding to stretching mode of $\mathrm{C}-\mathrm{N}$ triple bond. The same vibrations are computed by HF/6-311G $(d, p)$ and also shows good agreement with experimental data.

\subsection{Electronic absorption spectra and sensitized mechanism}

In order to understand the electronic transitions of 4-phenoxyphthalonitrile, TD-DFT calculations on electronic absorption spectra in vacuum and solvent were performed, and the results are shown in figure 5. It is observed that, for 4-phenoxyphthalonitrile, the absorption in the visible region is much weaker than that in the UV region. The calculated results have a red-shift. The results of TD-DFT have an appreciable red-shift, and the degree of red-shift in solvent is more significant than that in vacuum. The discrepancy between vacuum and solvent effects in TD-DFT calculations may result from two aspects. The first aspect is smaller gap of materials which induces smaller excited energies. The other is solvent effects. Experimental measurements of

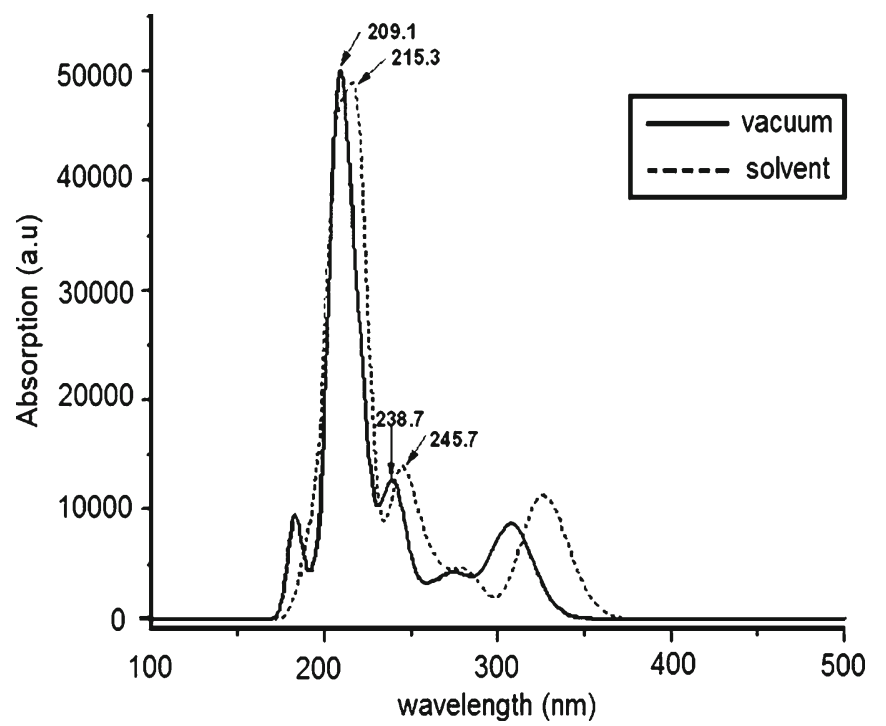

Figure 5. Calculated electronic absorption spectra of dye 4-phenoxyphthalonitrile. electronic absorptions are usually performed in solution. Solvent, especially polar solvent, could affect the geometry and electronic structure as well as the properties of the molecules through the long-range interaction between solute molecule and solvent molecule. For these reasons it is more difficult to make the TD-DFT calculation which is consistent quantitatively. Though the discrepancy exists, the TD-DFT calculations are capable of describing the spectral features of 4-phenoxyphthalonitrile because of the agreement of line shape and relative strength as compared with the vacuum and solvent.

The HOMO-LUMO gap of 4-phenoxyphthalonitrile in acetonitrile at B3LYP/6-31G $(\mathrm{d}, \mathrm{p})$ theory level is smaller than that in vacuum. This fact indicates that the solvent effects stabilize the frontier orbitals of 4-phenoxyphthalonitrile. So it induces the smaller intensities and red-shift of the absorption as compared with that in vacuum.

In order to obtain the microscopic information about the electronic transitions, the corresponding MO properties are checked. The absorption in visible and near-UV region is the most important region for photo-to-current conversion, so only the 20 lowest singlet/singlet transitions of the absorption band in visible and near-UV region for 4-phenoxyphthalonitrile is listed in table 5. The data of table 5 and figure 6 are based on the B3LYP/6-311G (d,p) results with solvent effects involved.

This indicates that the transitions are photoinduced charge-transfer processes, thus the excitations generate charge separated states, which should favour the electron injection from the excited dye to semiconductor surface.

The solar energy to electricity conversion efficiency $(\eta)$ under AM 1.5 white-light irradiation can be obtained from the following formula:

$$
\eta(\%)=\frac{J_{\mathrm{sc}}\left[\mathrm{mA} \mathrm{cm}^{-2}\right] V_{\mathrm{oc}}[\mathrm{V}] \mathrm{ff}}{I_{0}\left[\mathrm{~mW} \mathrm{~cm}^{-2}\right]} \times 100,
$$

where $I_{0}$ is the photon flux, $J_{\mathrm{sc}}$ the short-circuit photocurrent density and $V_{\text {oc }}$ the open-circuit photovoltage and ff represents the fill factor (Hara et al 2003a, b). At present, the $J_{\mathrm{sc}}$, the $V_{\mathrm{oc}}$ and the $f f$ are only obtained by the experiment, the relationship among these quantities and the electronic structure of dye is still unknown. The analytical relationship between $V_{\text {oc }}$ and $E_{\text {LUMO }}$ may exist. According to the sensitized mechanism (electron injected from the excited dyes to the semiconductor conduction band) and single-electron and single-state approximation, there is an energy relationship:

$$
\mathrm{e} V_{\mathrm{oc}}=E_{\mathrm{LUMO}}-E_{\mathrm{CB}},
$$

where $E_{\mathrm{CB}}$ is the energy of the semiconductor's conduction band edge. So the $V_{\text {oc }}$ may be obtained applying the following formula:

$$
V_{\mathrm{oc}}=\frac{\left(E_{\mathrm{LUMO}}-E_{\mathrm{CB}}\right)}{\mathrm{e}} .
$$


Table 5. Computed excitation energies, electronic transition configurations and oscillator strengths $(f)$ for the optical transitions of the absorption bands in visible and near-UV region for the dye 4-phenoxyphthalonitrile in acetonitrile.

\begin{tabular}{|c|c|c|c|c|}
\hline State & \multicolumn{2}{|c|}{$\begin{array}{l}\text { Configurations composition } \\
\text { (corresponding transition orbitals) }\end{array}$} & $\begin{array}{l}\text { Excitation energy } \\
(\mathrm{eV} / \mathrm{nm})\end{array}$ & $\begin{array}{l}\text { Oscillator } \\
\text { strength }(f)\end{array}$ \\
\hline 1 & $0.68365(57 \rightarrow 58)$ & & $3 \cdot 8065 / 325 \cdot 72$ & $0 \cdot 1567$ \\
\hline 2 & $\begin{array}{c}0.10446(54 \rightarrow 58) \\
-0.47544(57 \rightarrow 59)\end{array}$ & $0.50006(56 \rightarrow 58)$ & $4 \cdot 3461 / 285 \cdot 27$ & $0 \cdot 0020$ \\
\hline 3 & $0.49115(56 \rightarrow 58)$ & $0.48174(57 \rightarrow 59)$ & $4.4186 / 280 \cdot 59$ & 0.0569 \\
\hline 4 & $\begin{array}{c}-0.31756(54 \rightarrow 58) \\
0.52316(55 \rightarrow 58)\end{array}$ & $\begin{array}{l}-0 \cdot 16895(54 \rightarrow 59) \\
-0 \cdot 25930(55 \rightarrow 59)\end{array}$ & $4 \cdot 7770 / 259 \cdot 54$ & 0.0622 \\
\hline 5 & $\begin{array}{l}-0.21618(54 \rightarrow 58) \\
-0 \cdot 12427(55 \rightarrow 59) \\
-0.10712(57 \rightarrow 60)\end{array}$ & $\begin{array}{l}-0.22367(55 \rightarrow 58) \\
0.59152(56 \rightarrow 59)\end{array}$ & $5 \cdot 0105 / 247 \cdot 45$ & $0 \cdot 0228$ \\
\hline 6 & $\begin{array}{l}0 \cdot 35676(54 \rightarrow 58) \\
0 \cdot 33179(55 \rightarrow 59)\end{array}$ & $\begin{array}{l}0 \cdot 30876(55 \rightarrow 58) \\
0 \cdot 34177(56 \rightarrow 59)\end{array}$ & $5 \cdot 0881 / 243 \cdot 68$ & $0 \cdot 1538$ \\
\hline 7 & $\begin{array}{l}0 \cdot 14920(56 \rightarrow 59) \\
0 \cdot 34027(56 \rightarrow 61) \\
0 \cdot 11049(57 \rightarrow 61)\end{array}$ & $\begin{array}{c}-0 \cdot 11342(56 \rightarrow 60) \\
0.57540(57 \rightarrow 60)\end{array}$ & $5 \cdot 2940 / 234 \cdot 20$ & 0.0191 \\
\hline 8 & $\begin{array}{c}-0.28713(54 \rightarrow 58) \\
-0 \cdot 11101(56 \rightarrow 60) \\
0 \cdot 40077(57 \rightarrow 61)\end{array}$ & $\begin{array}{c}0 \cdot 39265(55 \rightarrow 59) \\
-0 \cdot 10158(57 \rightarrow 60)\end{array}$ & $5 \cdot 6653 / 218 \cdot 85$ & 0.5443 \\
\hline 9 & $\begin{array}{c}0 \cdot 20717(54 \rightarrow 58) \\
-0 \cdot 26362(56 \rightarrow 60) \\
0 \cdot 41253(57 \rightarrow 61)\end{array}$ & $\begin{array}{c}-0 \cdot 29384(55 \rightarrow 59) \\
-0 \cdot 13067(57 \rightarrow 60) \\
0 \cdot 16809(57 \rightarrow 62)\end{array}$ & $5 \cdot 9108 / 209 \cdot 76$ & $0 \cdot 2561$ \\
\hline 10 & $\begin{array}{c}0 \cdot 10411(53 \rightarrow 58) \\
-0 \cdot 14070(55 \rightarrow 60) \\
-0 \cdot 32288(57 \rightarrow 62)\end{array}$ & $\begin{array}{c}0 \cdot 49855(54 \rightarrow 59) \\
-0 \cdot 10846(56 \rightarrow 61)\end{array}$ & $6 \cdot 0547 / 204 \cdot 77$ & $0 \cdot 2187$ \\
\hline 11 & $\begin{array}{l}0 \cdot 25758(54 \rightarrow 59) \\
0 \cdot 16768(56 \rightarrow 60)\end{array}$ & $\begin{array}{l}-0 \cdot 17304(55 \rightarrow 62) \\
0 \cdot 54611(57 \rightarrow 62)\end{array}$ & $6 \cdot 0736 / 204 \cdot 14$ & $0 \cdot 1478$ \\
\hline 12 & $\begin{array}{l}-0.15741(52 \rightarrow 58) \\
-0 \cdot 19808(54 \rightarrow 59) \\
-0.10730(56 \rightarrow 61)\end{array}$ & $\begin{array}{c}0.55285(53 \rightarrow 58) \\
-0 \cdot 21054(55 \rightarrow 60)\end{array}$ & $6 \cdot 2321 / 198 \cdot 95$ & 0.0436 \\
\hline 13 & $\begin{array}{c}0 \cdot 10812(49 \rightarrow 58) \\
-0 \cdot 25252(52 \rightarrow 58) \\
0 \cdot 13790(54 \rightarrow 59) \\
0 \cdot 20780(56 \rightarrow 61) \\
-0 \cdot 12270(57 \rightarrow 61)\end{array}$ & $\begin{array}{c}0 \cdot 22684(51 \rightarrow 58) \\
0 \cdot 18261(53 \rightarrow 58) \\
0 \cdot 43416(55 \rightarrow 60) \\
-0 \cdot 13786(57 \rightarrow 60)\end{array}$ & $6 \cdot 2799 / 197 \cdot 43$ & $0 \cdot 0215$ \\
\hline 14 & $\begin{array}{c}0 \cdot 21900(49 \rightarrow 58) \\
-0 \cdot 33622(52 \rightarrow 58) \\
-0 \cdot 20452(55 \rightarrow 60)\end{array}$ & $\begin{array}{c}0.35392(51 \rightarrow 58) \\
-0.32290(53 \rightarrow 58) \\
-0 \cdot 10009(56 \rightarrow 61)\end{array}$ & $6 \cdot 3114 / 196 \cdot 44$ & $0 \cdot 0134$ \\
\hline 15 & $\begin{array}{c}0.55827(49 \rightarrow 58) \\
-0 \cdot 32012(51 \rightarrow 58) \\
-0 \cdot 11930(55 \rightarrow 60)\end{array}$ & $\begin{array}{c}0 \cdot 18047(50 \rightarrow 58) \\
-0 \cdot 10898(51 \rightarrow 59) \\
0 \cdot 37922(55 \rightarrow 61)\end{array}$ & $\begin{array}{l}6.3578 / 195 \cdot 01 \\
6.4560 / 192 \cdot 05\end{array}$ & $\begin{array}{l}0.0001 \\
0.0527\end{array}$ \\
\hline 16 & $\begin{array}{l}-0.11335(55 \rightarrow 62) \\
-0 \cdot 34160(56 \rightarrow 60) \\
-0.33806(57 \rightarrow 63)\end{array}$ & $\begin{array}{c}0 \cdot 10334(55 \rightarrow 63) \\
-0 \cdot 16842(56 \rightarrow 61)\end{array}$ & & \\
\hline 17 & $\begin{array}{c}0 \cdot 31846(55 \rightarrow 61) \\
-0 \cdot 14658(55 \rightarrow 63) \\
0 \cdot 14035(57 \rightarrow 62)\end{array}$ & $\begin{array}{c}0.18001(55 \rightarrow 62) \\
-0 \cdot 15738(56 \rightarrow 60) \\
0 \cdot 49710(57 \rightarrow 63)\end{array}$ & $6 \cdot 4953 / 190 \cdot 88$ & $0 \cdot 0265$ \\
\hline
\end{tabular}

It induces that the higher the $E_{\mathrm{LUMO}}$, the larger the $V_{\mathrm{oc}}$. The results of organic dye sensitizer JK16 and JK17 (Zhang et al 2009a, b, c), D-ST and D-SS also proved the tendency (Zhang et al 2009a) (JK16 : LUMO $=-2.73 \mathrm{eV}$, $V_{\mathrm{oc}}=0.74 \mathrm{~V} ; \mathrm{JK} 17: \mathrm{LUMO}=-2.87 \mathrm{eV}, V_{\mathrm{oc}}=0.67 \mathrm{~V}$;
D-SS : LUMO $=-2.91 \mathrm{eV}, V_{\text {oc }}=0.70 \mathrm{~V}$; D-ST : LUMO $\left.=-2.83 \mathrm{eV}, V_{\mathrm{oc}}=0.73 \mathrm{~V}\right)$. Certainly, this formula expects further test by experiment and theoretical calculation. The $J_{\mathrm{sc}}$ is determined by two processes, one is the rate of electron injection from the excited dyes to the 
Table 5. (contd.)

\begin{tabular}{|c|c|c|c|c|}
\hline \multirow{2}{*}{$\begin{array}{l}\text { State } \\
18\end{array}$} & \multicolumn{2}{|c|}{$\begin{array}{l}\text { Configurations composition } \\
\text { (corresponding transition orbitals) }\end{array}$} & $\begin{array}{c}\begin{array}{c}\text { Excitation energy } \\
(\mathrm{eV} / \mathrm{nm})\end{array} \\
6 \cdot 6793 / 185.63\end{array}$ & $\begin{array}{c}\begin{array}{c}\text { Oscillator } \\
\text { strength }(f)\end{array} \\
0.0099\end{array}$ \\
\hline & $\begin{array}{c}-0 \cdot 15119(52 \rightarrow 58) \\
0 \cdot 34270(54 \rightarrow 62) \\
0.43677(55 \rightarrow 62) \\
-0 \cdot 19421(57 \rightarrow 63)\end{array}$ & $\begin{array}{c}0 \cdot 14823(54 \rightarrow 61) \\
0 \cdot 12730(54 \rightarrow 63) \\
0 \cdot 13613(55 \rightarrow 63)\end{array}$ & $6 \cdot 6793 / 185 \cdot 63$ & 0.0099 \\
\hline 19 & $\begin{array}{c}-0 \cdot 11226(46 \rightarrow 58) \\
0 \cdot 24378(49 \rightarrow 58) \\
0 \cdot 32911(51 \rightarrow 58) \\
0 \cdot 10786(56 \rightarrow 62)\end{array}$ & $\begin{array}{c}-0 \cdot 13036(48 \rightarrow 58) \\
-0 \cdot 16625(50 \rightarrow 58) \\
0 \cdot 44728(52 \rightarrow 58)\end{array}$ & $6 \cdot 7321 / 184 \cdot 17$ & 0.0047 \\
\hline 20 & $\begin{array}{c}-0.11805(54 \rightarrow 60) \\
-0.20126(55 \rightarrow 62) \\
0.59139(56 \rightarrow 62) \\
0 \cdot 14179(57 \rightarrow 63)\end{array}$ & $\begin{array}{c}-0 \cdot 11034(55 \rightarrow 60) \\
0 \cdot 13316(56 \rightarrow 61) \\
-0 \cdot 10436(57 \rightarrow 62)\end{array}$ & $6 \cdot 7673 / 183 \cdot 21$ & $0 \cdot 0138$ \\
\hline
\end{tabular}

conduction band of semiconductor, and the other is the rate of redox between the excited dyes and electrolyte. Electrolyte effect on the redox processes is very complex, and it is not taken into account in the present calculations. This indicates that most of excited states of 4-phenoxyphthalonitrile have larger absorption coefficient, and then with shorter lifetime for the excited states. Hence it results in the higher electron injection rate which leads to the larger $J_{\mathrm{sc}}$ of 4-phenoxyphthalonitrile. On the basis of above analysis, it is clear that the 4-phenoxyphthalonitrile has better performance in DSSC.

HOMO-3

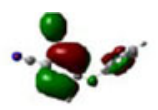

LUMO+3

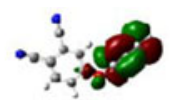

$-7.6622(\mathrm{eV})$

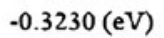

HOMO-2

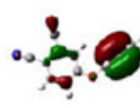

LUMO+2

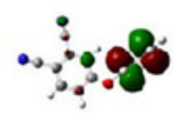

$-7.4225(\mathrm{eV})$

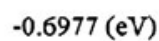

HOMO-1

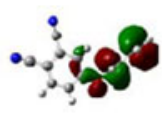

LUMO+1

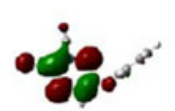

$-7.2015(\mathrm{eV})$

$-1.7554(\mathrm{eV})$

HOMO

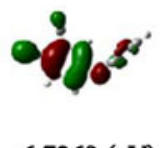

LUMO

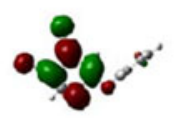

$-2.3323(\mathrm{eV})$

\section{Conclusions}

The geometries, electronic structures, polarizabilities and hyperpolarizabilities of dye 4-phenoxyphthalonitrile were studied by using $a b$ initio $\mathrm{HF}$ and density functional theory with hybrid functional B3LYP, and the UV-Vis spectra were investigated by using TD-DFT methods. The NBO results suggest that 4-phenoxyphthalonitrile is a (D-p-A) system. The calculated isotropic polarizability of 4-phenoxyphthalonitrile is -53.6627 a.u. The calculated polarizability anisotropy invariant of 4-phenoxyphthalonitrile is 171.8929 a.u. The hyperpolarizability of 4-phenoxyphthalonitrile is 5.3058 (in a.u). The strongest IR absorption for 4-phenoxyphthalonitrile corresponds to the vibrational mode 54 near about $1518 \mathrm{~cm}^{-1}$, which is corresponding to stretching mode of $\mathrm{C}=\mathrm{C}$ bonds. In the Raman spectrum, the strongest activity mode is the vibrational mode 60 near about $2339 \mathrm{~cm}^{-1}$, which is corresponding to stretching mode of $\mathrm{C} \equiv \mathrm{N}$ bond. The electronic absorption spectral features in visible and near-UV region were assigned based on the qualitative agreement to TD-DFT calculations. The absorptions were all ascribed to $\pi \rightarrow \pi^{*}$ transition. The three excited states with the lowest excited energies of 4-phenoxyphthalonitrile is photoinduced electron transfer processes that contributes sensitization of phototo-current conversion processes. The interfacial electron transfer between semiconductor $\mathrm{TiO}_{2}$ electrode and dye sensitizer 4-phenoxyphthalonitrile is electron injection process from excited dye as donor to the semiconductor conduction band. Based on the analysis of geometries, electronic structures and spectrum properties of 4-phenoxyphthalonitrile, the role of cyanine in phthalonitrile is as follows: it enlarged the distance between electron donor group and semiconductor surface and decreased the timescale of the electron injection rate, resulted in giving lower conversion efficiency. This indicates that the choice of the appropriate conjugate bridge in dye sensitizer is very important to improve the performance of DSSC.

Figure 6. Isodensity plots (isodensity contour $=0.02$ a.u.) of the frontier orbitals of 4-phenoxyphthalonitrile. 


\section{Acknowledgement}

This work was partly financially supported by University Grants Commission, Govt. of India, New Delhi, within the Major Research Project scheme under the approval-cumsanction No. F.No.34-5 2008 (SR) \& 34-1/TN/08.

\section{References}

Barone V and Cossi M 1998 J. Phys. Chem. A102 1995

Becke A D 1993 J. Chem. Phys. 985648

Campbell W M, Burrell A K, Officer D L and Jolley K W 2004 Coord. Chem. Rev. 2481363

Chiba Y, Islam A, Watanabe Y, Komiya R, Koide N and Han L 2006 Jpn. J. Appl. Phys. 45 L638

Christiansen O, Gauss J and Stanton J F 1999 Chem. Phys. Lett. 305 147

Cossi M, Rega N, Scalmani G and Barone V 2003 J. Comput. Chem. 24669

Datta A 2009 J. Phys. Chem. C113 3339

Datta A and Pati S K 2003 J. Chem. Phys. 1188420

Datta A and Pati S K 2004 J. Phys. Chem. A108 320

Datta A and Pati S K 2005a Chem. Eur. J. 114961

Datta A and Pati S K 2005b J. Mol. Struct. (Theochem) 75697

Datta A and Pati S K 2006 Chem. Soc. Rev. 351305

Datta A, Pati S K, Davis D and Sreekumar K 2005 J. Phys. Chem. A109 4112

Datta A, Terenziani F and Painelli A 2006 Chem. Phys. Chem. 72168

Edvinsson T et al 2007 J. Phys. Chem. C111 15137

Frisch M J et al 2003 Gaussian 03 (Pittsburgh, PA: Gaussian, Inc.)

Gratzel M 2001 Nature 414338

Hagberg D P, Edvinsson T, Marinado T, Boschloo G, Hagfeldt A and Sun L 2006 Chem. Commun. 2245

Hara K, Kurashige M, Dan-oh Y, Kasada C, Shinpo A, Suga S, Sayama K and Arakawa H 2003a New J. Chem. 27783

Hara K et al 2003b J. Phys. Chem. B107 597

Horiuchi T, Miura H, Sumioka K and Uchida S 2004 J. Am. Chem. Soc. 12612218

Ito $\mathrm{S}$ et al 2008 Chem. Commun. 5194

Kim S et al 2006 J. Am. Chem. Soc. 12816701

Kitamura T, Ikeda M, Shigaki K, Inoue T, Anderson N A, Ai X, Lian T and Yanagida S 2004 Chem. Mater. 161806
Koumura N, Wang Z S, Mori S, Miyashita M, Suzuki E and Hara K 2006 J. Am. Chem. Soc. 12814256

Lee C, Yang W and Parr R G 1988 Phys. Rev. B37 785

Li S L, Jiang K J, Shao K F and Yang L M 2006 Chem. Commun. 2792

Liang M, Xu W, Cai F, Chen P, Peng B, Chen J and Li Z 2007 J. Phys. Chem. C111 44652

Lin J T, Chen P C, Yen Y S, Hsu Y C, Chou H H and Yeh M C P 2009 Org. Lett. 1197

Lundqvist M J, Nilsing M, Persson P and Lunell S 2006 Int. J. Quantum Chem. 1063214

Miehlich B, Savin A, Stoll H and Preuss H 1989 Chem. Phys. Lett. 157200

Nazeeruddin M K et al 2005 J. Am. Chem. Soc. 12716835

Park N G and Kim K 2008 Phys. Status Solidi A205 1895

Regan B O and Gratzel M 1991 Nature 353737

Seidl A, Gorling A, Vogl P, Majewski J A and Levy M 1996 Phys. Rev. B53 3764

Shi D, Pootrakuchote N, Yi Z, Xu M, Zakeeruddin S M, Gratzel M and Wang P 2008 J. Phys. Chem. C112 17478

Sun Y, Chen X, Sun L, Guo X and Lu W 2003 Chem. Phys. Lett. 381397

Thomas K R J, Lin J T, Hsu Y C and Ho K C 2005 Chem. Commun. 4098

Wang M et al 2008 Adv. Mater. 204460

Wang Z S, Huang Y Y, Huang C H, Zheng J, Cheng H M and Tian S J 2000 Synth. Met. 14201

Wang Z S, Cui Y, Hara K, Dan-oh Y, Kasada C and Shinpo A 2007 Adv. Mater. 191138

Waston D F and Meyer G J 2005 Annu. Rev. Phys. Chem. 56119

Xu M et al 2009 J. Phys. Chem. C113 2966

Xu W, Peng B, Chen J, Liang M and Cai F 2008 J. Phys. Chem. C112 874

Zhang C R, Chen H S and Wang G H 2004 Chem. Res. Chin. U. 20640

Zhang X H, Li C, Wang W B, Cheng X X, Wang X S and Zhang B W 2007 J. Mater. Chem. 17642

Zhang C R, Liu Z J, Chen Y H, Chen H S, Wu Y Z and Yuan L H 2009a J. Mol. Struct. (THEOCHEM) 89986

Zhang C R, Wu Y Z, Chen Y H and Chen H S 2009b Acta Phys. Chim. Sin. 2553

Zhang G, Bai Y, Li R, Shi D, Wenger S, Zakeeruddin S M, Gratzel $\mathrm{M}$ and Wang P 2009c Energy Environ. Sci. 292

Zhou G, Pschirer N, Schoneboom J C, Eickemeyer F, Baumgarten M and Mullen K 2008 Chem. Mater. 201808 